\title{
Etiological characteristic and risk factors of ESBL- Urinary Tract Infections: A nomogram had been built and Urologist should act in time
}

\section{Yi Wei}

Chongqing Medical University Affiliated Children's Hospital

\section{Chengjun Yu}

Chongqing Medical University Affiliated Children's Hospital

Tianxing Zhao

Chongqing Medical University Affiliated Children's Hospital

Tao Lin

Chongqing Medical University Affiliated Children's Hospital

\section{Dawei He}

Chongqing Medical University Affiliated Children's Hospital

Sheng-de Wu ( $\sim$ shengdewu@yeah.net)

Department of Urology, Children's Hospital of Chongqing Medical University, Chongqing

\section{Guanghui Wei}

Chongqing Medical University Affiliated Children's Hospital

\section{Research}

Keywords: Urinary tract infection (UTI), Beta-Lactamase (ESBL)-producing bacteria, Urological abnormalities, Neurologic disorder, Risk factor

Posted Date: May 29th, 2020

DOI: https://doi.org/10.21203/rs.3.rs-31324/v1

License: (c) (1) This work is licensed under a Creative Commons Attribution 4.0 International License. Read Full License 


\section{Abstract}

Background: The presence of urinary tract infection (UTI) due to Extended-Spectrum Beta-Lactamase (ESBL)-producing bacteria is reported increased. Aim to study the most frequent uropathogens and the antibiotic susceptibility patterns of them in children and identify whether urodynamic change, underlying neurologic disorders and undernourishment were independent risk factors for ESBL positive UTI which is unclear.

Methods: We retrospectively reviewed microbial etiologies and antimicrobial resistance among patients experiencing UTI events in the urology ward of Chongqing Medical University Afflicated Chlidren's Hospital from January 1994 and December 2019. All strains were cultured and identified by the Clinical Microbiology Laboratory.

Results: A total of 854 patients with UTI over a 26-years period were evaluated and Escherichia coli was the most common pathogen. During the study period, the proportion of UTI cases attributed to Enterococci increased significantly. Susceptibilities to carbapenems and amikacin decreased significantly, indicating increased antibiotic resistance of pathogens associated with UTI. Interestingly, the susceptibilities to piperacillin/tazobactam have increased. $72.64 \%$ were caused by ESBL bacteria and ESBL-producing bacteria increased significantly. ESBL (+) and ESBL (-) UTI were compared and there were no significant differences in the clinical presentation between gender, side of the lesion and urodynamic results. Significant potential risk factors of ESBL-UTI were presence of congenital urological abnormalities, vesicoureteral reflux, neurologic disorder, age $<12$ months, fever and previous use of antibiotics in the last 3 months. On logistic regression analysis, underlying neurologic disorder $(\mathrm{OR}=8, \mathrm{Cl}$ 1.845-34.695) and history of previous antibiotics administration in the last 3 months $(\mathrm{OR}=4.764, \mathrm{Cl}$ 3.114-7.289) were identified as an independent significant risk factor for ESBL- UTI. The nomogram generated was well calibrated for all predictions of ESBL+ probability, and the accuracy of the model nomogram measured by Harrell's C statistic (C-index) was 0.741 .

Conclusions: The current situation of multiple bacterial antibiotic resistance has become a worrisome issue in UTI, and urologist should act timely. Our data will greatly assist physicians recognizing the risk factors of ESBL-UTI and optimising antibiotic use.

\section{Introducing}

Urinary tract infection (UTI) is the most common infection among young infants and children ${ }^{[1]}$. They can be associated with long-term complications such as renal scarring and chronic renal failure whereby early treatment is needed ${ }^{[2]}$.

UTIs are most frequently due to Enterobacteriaceae, mainly Escherichia coli ${ }^{[3,4]}$. However, Escherichia coli species have variable antimicrobial resistance mechanisms which may include the production of extended-spectrum- $\beta$-lactamase (ESBL). ESBL are plasmid mediated enzymes that degrade penicillins, cephalosporins but spare cephamycins (cefoxitin, cefotetan), moxalactam and carbapenems and are 
mostly produced by Enterobacteriaceae ${ }^{[5]}$. A recent systematic review and meta-analysis has shown a $14 \%$ pooled prevalence of pediatric UTI caused by ESBL producing enterobacteriaceae in different countries ${ }^{[6]}$. The emergence of ESBL-producing Enterobacteriaceae in pediatric urinary tract infections presents a serious threat to public health because no oral antibiotic as first-line treatment is regularly active against ESBL UTI and there are few intravenous options.

Thus, knowledge of the microorganisms involved in UTI and risk factors of urinary tract infections caused by ESBL-producing bacteria in children is important ${ }^{[7,8]}$. Several limited studies have described the risk factors of ESBL UTI in children ${ }^{[9-12]}$. Among the risk factors were underlying disease, patients with vesicoureteric reflux, UTI prophylaxis, previous UTI, hospitalization within the last three months, recent antibiotic use and high UTI recurrence rate.

In a systematic review and meta-analysis, 26 cross-sectional and 8 case-control studies reporting on UTI prevalence in malnourished children was included, and they found that UTI is more prevalent in malnourished children than in their well-nourished counterparts ${ }^{[13]}$. Another study included 402 malnourished children in Tanzania and data showed that more than $37 \%$ of UTI isolates were exhibiting extended spectrum beta lactamase (ESBL) phenotype ${ }^{[14]}$. A retrospective study reviewed microbial etiologies and antimicrobial resistance among patients experiencing UTI events in the neurology ward and ESBL-producing K. pneumoniae increased significantly ${ }^{[15]}$. To our knowledge, until now, the most frequent uropathogens and the antibiotic susceptibility patterns of them in children with congenital urological malformations is still largely unknown, whether fever, urodynamic abnormality, undernourishment and underlying neurologic disorders were independent risk factors for ESBL positive UTI is also unclear. Furthermore, using multivariable logistic regression coefficients to construct a corresponding nomogram and predict the probability of ESBL (+) UTI is needed.

Recently, we noticed a substantial increase in the incidence of ESBL UTI in our children at our institution. This prompted us to investigate the risk factors of acquisition of ESBL UTI and study their clinical characteristics, antimicrobial resistance in comparison to children with non- ESBL UTI. Determination of these risk factors will help us in choosing the appropriate initial antibiotic, preventing sub-optimal treatment and antimicrobial resistance.

\section{Methods}

This is a retrospective case control study conducted at Chongqing Medical University Afflicated Chlidren's Hospital. Clinical data of patients with UTI were reviewed via an electronic medical records system for patients who were admitted to the urology ward of the Chongqing Medical University Afflicated Chlidren's Hospital in Chongqing, China between January 1994 and December 2019.

The age, gender, fever, side of the lesion, underlying renal anomalies, vesicoureteral reflux, urodynamic change, underlaying neurologic disorders, previous UTI in the last three months, previous antibiotics 
administration in the last three months, nutritional status, microbial cultures, antibiotic sensitivity of a urinary specimen and main diagnosis during admission to the urology ward were obtained.

Urine specimens were obtained according to the American Academy of Pediatrics (AAP) guidelines by bladder catheterization in young children and by mid-stream sampling in toilet trained children ${ }^{[16]}$. One urine culture was used for each patient. Mixed growths of cultures were excluded.

Positive urine culture was more than 50,000 colony-forming units/ml. UTI was defined as abnormal urine analysis and a positive urine culture according to the American Academy of Pediatrics guidelines. Identification and susceptibility profiling of all bacteria from positive urine cultures were determined by using an automated susceptibility system VITEK 2 (Bio Merieux, Marcy I Etoile, France) according to the Clinical and Laboratory Standard Institute guidelines. Asymptomatic bacteriuria or no identified bacterium in an urinary culture was excluded from the study.

Statistical analysis: A descriptive statistical analysis was performed, and differences in susceptibility rates were analyzed using contingency tables with Fisher's exact test. The Cochran-Mantel-Haenszel test was used to assess trends associated with the annual spectrum of microbes and antibiotic susceptibilities of UTI-associated pathogens. Logistic regression was used to analyze the evolution of antibiotic activity against isolated bacteria in UTIs over the 26-year study period. A predictive model (nomogram) was also built using statistical software R (The R Foundation for Statistical Computing, Vienna, Austria). SPSS 19 was used to analyze the collected data. P-values $<0.05$ was considered significant in all statistical analyses.

Ethics Statements: This was a non-interventional study involving only routine procedures. Biological material was used solely for standard UTI diagnosis prescribed by physicians, and there was no additional sampling or modification of the standard laboratory protocol. Data analyses were carried out using an anonymous database. For these reasons, no approval was required according to national guidelines. Permission to access and use the data was granted by the Unit for Clinical Management of Chongqing Medical university.

\section{Results}

\section{Clinical characteristics of urological patients with UTI}

A total of 854 children with UTI over 26 years were identified included $614(71.90 \%)$ patients with congenital urological malformations (Hydronephrosis 305, Vesicoureteral reflux 136, Neurogenic bladder 44, Duplex kidney 41, Bladder diverticulum 9, Posterior urethral valves 17, Intravesical ureterocele 34 , Prostatic utricle 7, Urethral diverticulum 3, Phimosis 2, Renal hypoplasia 8, Congenital Megaureter 2, Exstrophy of bladder 6) and 240 patients with no underlying disease.

\section{The microbial spectrum of UTI from a urinary culture}


The microbial spectrum of UTI from a urinary culture is shown in Figure 1. A. Over the 26 year study period, the most common pathogen associated with UTI was Escherichia coli (295 cases, 34.54\% of all isolates; range from $20.00 \%$ to $45.16 \%$ ), followed by Enterococcus species (Enterococcus faecium 99, Enterococcus faecalis 46,145 cases, $16.98 \%$; range, from $6.67 \%$ to $37.50 \%$ ), Klebsiella pneumoniae (105 cases, $12.30 \%$; range from $5.80 \%$ to $21.88 \%$ ), Other Enterobacter spp (Enterobacter cloacae 48 , Enterobacter aerogenes 11, 59 cases, 6.90\%), Pseudomonas aeruginosa (58 cases, 6.79\%), Proteus spp (Proteus mirabilis 27, Proteus vulgaris 11, 38 cases, 4.45\%) and Staphylococci (Staphylococcus epidermidis 11 , Staphylococcus aureus 17 , Staphylococcus haemolyticus 7 , staphylococcus saprophyticus 3, Staphylococcus caprae 1, 39 cases, 4.57\%). Other bacteria (Citrobacter spp; Serratia marcescens; Morganella morganii; and Stenotrophomonas maltophilia) accounted for only $13.47 \%$ $(115 / 854)$ of all isolates. During the study period, the proportion of UTI cases attributed to Enterococci increased significantly $(p=0.000)$; however, the overall proportion of E. coli $(p=0.753), K$. pneumoniae $(p=$ $0.071)$ and $P$. aeruginosa $(p=0.198)$ did not change.

The prevalence of Enterococcus in children with urological abnormality was higher than that of children with no urological abnormality (Fig. 1. B).

\section{Trends of antibiotics susceptibility in pathogens involved in UTI}

The antibiotics administered to treat UTIs were reviewed. Piperacillin/tazobactam was the most frequently prescribed antibiotics for UTI ( 505 cases, $59.13 \%$ ), followed by latamoxef ( 179 cases $20.96 \%$ ), the 3rd generation cephalosporins (Ceftazidime 39, Cefotaxime 22, Cefoperazone 17, Cefixime 4, Ceftriaxone 12 cases, total 94 cases 33.2\%), quinolone (26 cases, 3.04\%), carbapenems (44cases, 5.15\%) and vancomycin (22 cases, $2.58 \%$ ).

The trend of susceptibility to common antibiotics in UTI pathogens is shown in Figure 2. The 26-year overall susceptibility of UTI cases to individual antibiotics was as follows: $59.72 \%$ ( 903 susceptible over 1512 ) to quinolones (levofloxacin and ciprofloxacin), 29.13\% (60 susceptible over 206) to the 2nd generation cephalosporins, $40.64 \%$ (608 susceptible over 1496) to the 3rd generation cephalosporins, $71.35 \%$ (411 susceptible over 576 ) to piperacillin/tazobactam, $30.99 \%$ (159 susceptible over 513 ) to the amoxicillin/clavulanic acid $99.46 \%$ (185 susceptible over 186 ) to vancomycin, $90.79 \%$ (1104 susceptible over 1216) to carbapenems (imipenem, meropenem, and ertapenem), 49.65 (140 susceptible over 282) to nitrofurantoin, $77.76 \%$ (577 susceptible over 742 ) to amikacin and $46.51 \%$ (366 susceptible over 787 ) to gentamicin.

Over the last 26 years, the susceptibilities to quinolones ( $p$-value $=0.833)$, vancomycin ( $p$-value $=0.361)$, the 3 rd generation cephalosporins ( $p$-value $=0.172)$, gentamicin $(p$-value $=0.752)$ and nitrofurantoin $(p-$ value $=0.246)$ were unchanged. The susceptibilities to carbapenems ( $p$-value $=0.02)$ and amikacin $(p-$ value $=0.004$ ) decreased significantly. Interestingly, the susceptibilities to piperacillin/tazobactam have increased in UTI-associated pathogens ( $p$-value $=0.039)$. (Fig 2. B). 
Antibiotic Susceptibility of the Isolated Bacteria (E. coli, K. pneumoniae, Pseudomonas aeruginosa, Enterobacter cloacae, and Enterococcus species).

Figure. 3 exhibits the antimicrobial susceptibilities of the most frequent microorganisms, E. coli, K. pneumoniae, Pseudomonas aeruginosa, Enterobacter cloacae, and Enterococcus species. The percentages of susceptibility to the different study antibiotics were highly variable in the different species. E. coli showed high susceptibility to piperacillin/tazobactam, nitrofurantoin, amikacin, carbapenems, and vancomycin. K. pneumoniae showed high susceptibility to quinolones, carbapenems, and vancomycin; however, resistance to piperacillin/tazobactam and nitrofurantoin was recorded in at least $30 \%-40 \%$ of K. pneumoniae isolates in these years. Enterobacter cloacae were also detected high resistance to piperacillin/tazobactam with resistance rates $>45 \%$.

Pseudomonas aeruginosa showed high susceptibility to quinolones, piperacillin/tazobactam, carbapenems, and vancomycin, with annual resistance rates $<10 \%$. Enterococcus species showed high susceptibility to carbapenems and vancomycin, whereas resistance to gentamicin, amikacin, and quinolones in most years.

\section{The prevalence of ESBL (+) pathogens in UTI.}

We analyzed whether the prevalence of antibiotics-resistant pathogens in UTI had increased over the past 25 years. In our study of hospitalized patients with UTI over a 26 years interval $72.64 \%$ were ESBL producers which is a high percentage. Interestingly, the presence of ESBL-producing UTI increased significantly over the past 25 years $(p=0.003)$. Among the isolated bacteria, the presence of ESBLproducing pathogens in $E$. coli increased significantly $(p=0.021)$. However, ESBL in K. pneumoniae did not change over the last 25 years ( $p$-value $=0.482$ ).

The 26-year overall susceptibility of UTI cases to individual antibiotics was as follows: $52.90 \%$ to quinolones (levofloxacin and ciprofloxacin), $11.22 \%$ to the 2 nd generation cephalosporins, $15.18 \%$ to the 3rd generation cephalosporins, $59.94 \%$ to piperacillin/tazobactam, $21.86 \%$ to amoxicillin/clavulanic acid, $100 \%$ to vancomycin, $90.10 \%$ to carbapenems (e.g. imipenem, meropenem and ertapenem), $54.76 \%$ to nitrofurantoin, $91.90 \%$ to amikacin and $38.95 \%$ to gentamicin.

\section{Risk factors for acquisition of ESBL producing UTI.}

Regarding the risk factors, the following were statistically significantly associated with ESBL- UTI: presence of congenital urological abnormalities ( $p$-value $=0.002)$, presence of vesicoureteral reflux ( $p$ value $=0.041)$, presence of neurologic disorder ( $p$-value $=0.011)$, age $<12$ months ( $p$-value $=0.002)$, fever $(p$-value $=0.006)$ and previous use of antibiotics in the last 3 months $(p$-value $=0.000)($ Table 1$)$.

Forward logistic regression analysis identified underlying neurologic disorder $(\mathrm{OR}=8, \mathrm{Cl} 1.845-34.695, \mathrm{p}$ value of 0.005$)$ and history of previous antibiotics administration in the last 3 months $(O R=4.764, \mathrm{Cl}$ 3.114-7.289, p-value of 0.000) as independent risk factors for ESBL positive UTI (Table 2). 


\section{Diagnostic use of nomogram.}

To predict the probability of ESBL (+) UTI, we also used multivariable logistic regression coefficients to construct a corresponding nomogram and calibration plot for these data (Figure 6). The nomogram generated was well calibrated for all predictions of ESBL+ probability, and the accuracy of the model nomogram measured by Harrell's C statistic (C-index) was 0.741 .

In the final points-based nomogram (Figure 6. A), significant predictors are located on the left side, tailing with their respective scales on the right. Each scale position has corresponding points located on the "point" scale. The sum of all points for each variable was used to calculate the "Total Points". Each "Total Points" represents the different probability to be a ESBL (+) UTI. For example, in a 3-months-old patient, who had previous UTI, fever, a history of antibiotics administration in the last 3 months, renal anomalies and vesicoureteral reflux. For this patient, the "Total Points" $=14(<12$ months) +0 (previous UTI) +11 (fever) +95 (antibiotics administration) +16 (renal anomalies) +4 (vesicoureteral reflux $)=140$. The probability of ESBL $(+)$ for this patient was $88.9 \%$. The calibration plots (Figure 6. B) suggested, in general, the nomogram was well calibrated for all predictions of ESBL $(+)$ probability.

\section{Discussion}

This study describes trends in the microbial etiology of and antimicrobial resistance in UTI during19942019 in the urology ward in the Chongqing Medical University Affiliated Children's Hospital (Chong Qing, China). Within this geographic area, we compared data on 854 urine samples from the pediatric population.

In our cases, E. coli was the most frequently bacterium isolated in urine samples of patients with UTI (34.54\%), followed by Enterococcus species (16.98\%) and Klebsiella pneumoniae (12.30\%). These data are consistent with previous findings that E.coli is the most common pathogen associated with UTI ${ }^{[17-19]}$. K. pneumoniae was described the second most frequently isolated pathogen in other studies ${ }^{[15]}$. However, the frequency of Enterococcus. spp in our region was higher than that of K. pneumoniae. This difference may be because part of UTIs in this study were hospital-acquired (291 cases, 34.07\%). In hospital acquired UTI, Enterococcus species was more common, whereas in community-acquired UTI, K. pneumoniae was more common among the pathogens ${ }^{[20]}$. On the other hand, the prevalence of Enterococcus species is reported higher in children with underlying urological malformations ${ }^{[21]}$. In our study, $71.90 \%$ children were identified with urological malformations and the prevalence of Enterococcus in these children was higher than that of children with no urological abnormality ( $28.75 \%$ vs $17.75 \%$ ). Hence, Enterococcus species was more frequent in our study, although this is not necessarily the case in different geographic areas and country. Moreover, the proportion of UTI cases attributed to Enterococci increased significantly while that of E. coli, K. pneumoniae and P. aeruginosa did not change. Thus, therapeutic recommendations must take into account the low activity of cephalosporins and aminoglycosides against Enterococcus spp. 
The antibiotics administered to treat UTI were reviewed. Piperacillin/tazobactam was the most frequently prescribed antibiotic for UTI, followed by latamoxef, the 3rd generation cephalosporins and quinolone. However, whether these antibiotics were susceptible to the isolated bacteria is largely unknown. In our study, the 26-year overall susceptibility of UTI cases to piperacillin/tazobactam was $71.35 \%$, while that of the 3 rd generation cephalosporins and quinolone was only $40.64 \%$ and $59.72 \%$. Over the last 26 years, the susceptibilities to quinolone, the 3rd generation cephalosporins, gentamicin and nitrofurantoin were unchanged. Interestingly, the susceptibilities to piperacillin/tazobactam have significantly increased in UTI-associated pathogens. In agreement with our results, various studies of pediatric populations have reported a high susceptibility to piperacillin-tazobactam $[20,22,23]$. On the contrary, Hye-Rim found that the susceptibilities to piperacillin/tazobactam have decreased in UTI-associated pathogens from 2007$2016^{[15]}$. This difference may be because of the proportion of children with underlying urological malformations. The risk of UTI is increased among children with an underlying urological abnormality such as vesicoureteral reflux, constipation, and voiding dysfunction ${ }^{[21]}$. The prevalence of "atypical" bacteria is also higher. Considering different isolated bacteria, the percentages of susceptibility to the different study antibiotics were highly variable ${ }^{[24]}$. Furthermore, isolated pathogens from different country and region should also be considered.

Considering species separately, E. coli showed high susceptibility to piperacillin/tazobactam and nitrofurantoin. High resistance to gentamicin, the 3rd generation cephalosporins and nitrofurantoin was recorded in at least $30 \%-40 \%$ of $\mathrm{K}$. pneumoniae isolates in these years. Pseudomonas aeruginosa showed high susceptibility to quinolones and piperacillin/tazobactam, with annual resistance rates $<15 \%$. Enterococcus species showed high susceptibility to carbapenems and vancomycin, whereas resistance to gentamicin, amikacin and quinolones. Hence, although various agents are valid options, our study recommend piperacillin/tazobactam as the first choice, while the 3rd generation cephalosporins and quinolone would therefore not be suitable for empiric UTI treatment in southwest region, China.

Extended spectrum beta-lactamases (ESBLs) are enzymes capable of hydrolysing penicillins, broadspectrum cephalosporins and monobactams, and are generally derived from TEM and SHV-type enzymes. Antibiotics-resistant bacteria especially the ESBL- producing bacteria are increasing worldwide and are a serious problem in infection control ${ }^{[25,26]}$.To our knowledge this is the first pediatric study from Chongqing that assess the risk factors for the emergence of ESBL-producing bacteria.

Although the prevalence of ESBLs is not known, it is clearly increasing. In our study of hospitalized patients with UTI over a 26 years interval $72.64 \%$ were ESBL producers which is a high percentage. Hanna-Wakim et al had evaluated hospitalized children over ten years and reported an ESBL positivity rate of $15.5 \%{ }^{[3]}$. A recent systematic review and metanalysis has shown a pooled prevalence of pediatric ESBL UTI of $5 \%$ in eastern Mediterranean studies compared to an overall prevalence of $14 \%$ in all other countries $^{[6]}$. However, it has reached to $46 \%$ and $49.3 \%$ in a cohort study from Turkey and Jordan ${ }^{[9,27]}$. Considering all species together, we did find a statistically significant increase in ESBL-producing 
pathogens. There was also an observed increase in the proportion of ESBL producing pathogens among E. coli UTI.

This high percentage and the trend could be attributed to the fact that our study included only hospitalized children in a third-grade class A hospital where more complicated cases were admitted. Furthermore, some of the patients were hospitalized and treated with antibiotics for a long period because of UTI before transferring to our hospital. The high proportion of children with an underlying urological abnormality would also promote ESBL presence increasing.

ESBL-producing strains are particularly important as they are resistant to all penicillin, to aztreonam and the majority cephalosporins (including third and fourth generation agents), furthermore, they are often cross-resistant to trimethoprim/ sulfamethoxazole and quinolones. Thus, early identification of ESBL production is important in terms of appropriate treatment and effective infection control. While finding the risk factors of ESBL-producing would contribute to this item.

Previous studies have shown that hospitalization and use of antibiotics in the last 3 months, history of recurrent UTI, and presence of renal anomalies were important risk factors for ESBL-UT[ [25, 28]. Neurological patients are increasingly vulnerable to a UTI due to the presence of a neurogenic bladder or maintenance of a urinary catheter. Previous studies also show the proportion of ESBL-producing UTI is increasing in neurological patients ${ }^{[15,29]}$. However, whether neurologic disorder (e.g. tethered cord syndrome, meningitis, hypoxic ischemic encephalopathy, invisible spina bifida) and urodynamic change were risk factors for ESBL-UTI is still unknown. Studies also reported that the prevalence of UTI and ESBL (+) UTI was higher in malnourished children ${ }^{[13,14]}$. Whether undernourishment was independent risk factors for ESBL positive UTI is also unclear.

Hence, our study identified age at diagnosis as $<12$ months, fever, presence of urological malformation, vesicoureteral reflux, urodynamic change, neurologic disorder, undernourishment, previous use of antibiotics in the last 3 months and previous UTI in the last 3 months as predictors of acquisition of ESBL-UTI.

It is suggested that children with underlying neurologic disorder (e.g. tethered cord syndrome, meningitis, hypoxic ischemic encephalopathy, invisible spina bifida) was identified as the most significant risk factor by for ESBL-UTI in our study. This was supported by other studies performed on the risk factors for UTIs by ESBL-producing bacteria in neurological patients. Although they didn't perform logistic regression analysis to identified neurological disorders as independent risk factors for ESBL positive UTI, there was an observed increase in the proportion of ESBL producing pathogens among children with neurological abnormality ${ }^{[15,30]}$. Therefore, it is necessary to perform a detailed history-taking and physical examination among UTI children to identify the presence of underlying neurological disease.

Previous studies have identified previous antibiotic use and children with recurrent UTI as independent factors for ESBL UT ${ }^{[31]}$. This trend was also observed in our study. Forward logistic regression analysis 
identified history of previous antibiotics administration in the last 3 months as independent risk factors for ESBL positive UTI (OR =4.726, Cl 3.090-7.229, p-value= 0.000). Although the exact mechanisms underlying this association are not clear, we considered that previous antibiotics administration will lead to colonization of these children with ESBL organisms predisposing them to future UTI. This might be also explained by the increasing rate of fecal colonization with these resistant bacteria in healthy carriers $^{[15,31]}$.

In our cohort of patients, we observed a trend regarding a higher incidence of ESBL cases in children less than year (55.8\% of ESBL cases) which is similar to two studies from Turkey and one study from Jordan $^{[9]}$. In our study, $40 \%$ of the children less than one year of age and $38.75 \%$ of those more than one year of age had ESBL producing bacteria that were resistant to amoxicillin/clavulanic in $58.70 \%$, to nitrofurantoin in $22.62 \%$, to quinolones in $43.14 \%$, and gentamicin in $59.67 \%$

All children with known mechanical obstruction (posterior urethral valves, strictures) and unctional obstruction (lower urinary tract dysfunction of either neurogenic or non-neurogenic origin and dilating vesicoureteral reflux) are considered to be related to ESBL (+) UTI. ${ }^{[22,26,32]}$. This trend was also observed in our study. Presence of congenital urological anomalies, presence of vesicoureteral reflux, fever and previous UTI in the last three months were statistically significantly associated with ESBL-UTI. Although these factors were not identified as independent risk factors for ESBL (+) UTI with conditional logistic regression analysis. These patients require hospitalisation and parenteral antibiotics. Prompt anatomical evaluation of the urinary tract is critical to exclude the presence of significant urological or neurological abnormalities. If mechanical or functional abnormalities are present, adequate drainage of the infected urinary tract is necessary.

In a systematic review and meta-analysis, 26 cross-sectional and 8 case-control studies reporting on UTI prevalence in malnourished children was included, and they found that UTI is more prevalent in malnourished children than in their well-nourished counterparts ${ }^{[13]}$. Another study included 402 malnourished children in Tanzania and data showed that more than $37 \%$ of UTI isolates were exhibiting extended spectrum beta lactamase (ESBL) phenotype ${ }^{[14]}$. Although $83.33 \%$ of the nourished children were ESBL (+) in our study, this factor was not significantly associated with ESBL- UTI.

In the present series, there were no clinical significant factors as gender, side of the lesion and with urodynamic abnormality, in regards to the acquisition of ESBL-UTI in these infants. Thus, these data suggest three possible points of intervention: 1 . The policy of restricted indications for administrating antibiotics could reduce the incidence of resistance. 2.Optimise antibiotic use among young children with urological anomalies, vesicoureteral reflux and recurrent UTI. 3. Choosing broad-spectrum antibiotics, including carbapenems, as empirical antibiotics for UTI among children with neurological disease and used antibiotic in the last three months.

Guidelines from the National Institute for Health and Clinical Excellence and American Academy of Pediatrics differ from each other in terms of the diagnostic algorithm to be followed. Treatment of ESBL- 
UTI and antibiotic prophylaxis for prevention of recurrent UTI are also areas of considerable debate. At present, the 2nd or 3rd generation cephalosporins and amoxicillin/clavulanic acid are recommended the first choice for empirical treatment ${ }^{[33,34]}$. Some studies have demonstrated that once daily parenteral administration of gentamicin or ceftriaxone in a day treatment center is safe, effective and cost-effective in children with $\mathrm{UTT}^{[35,36]}$. However, the major concern in our study is the high resistance to 2 nd generation cephalosporins ( $>80 \%)$, 3rd generation cephalosporins ( $>80 \%)$ and the amoxicillin/clavulanic acid $(>55 \%)$. Similarly, excluding amikacin, carbapenems, vancomycin and piperacillin/tazobactam, antibiotics with sensitivity higher than $50 \%$ to ESBL-producing pathogens were absent in our study.

Edlin et al ${ }^{[37]}$ found that although widely used, trimethoprim-sulfamethoxazole is a poor empirical choice for pediatric urinary tract infections in many areas due to high resistance rates. First-generation cephalosporins and nitrofurantoin are appropriate narrow-spectrum alternatives given their low resistance rates. In agreement with their study, nitrofurantoin is an appropriate choose with resistance rates $<25 \%$. Therefore, overconsumption in low-risk settings should be avoided and pediatricians should be aware about the specific treatment options. Any recommendation about (initial) antibiotic treatment should be regularly updated and adapted to local resistance profiles.

This study has some limitations. We reviewed limited data collected from a single medical center, so it may not reflect the national status of microbial etiology of and antibiotics resistance in UTI. However, this study is very important as no previous studies were characterizing long-term surveillance data for UT Is in all urological patients in a tertiary referral hospital in China. And since this was a retrospective study and depended on medical records, some UTI events may have been missed due to insufficient medical records. So, the nationwide surveillance program of microbial etiology and antibiotics resistance in infectious diseases is needed.

\section{Conclusion}

In conclusion, there is an increasing presence of ESBL-UTI and urologist should act timely. Children with underlying neurologic disorders and previous use of antibiotics are at increased risk for these infections. Identifying these risk factors and developing a nomogram for predictions of ESBL+ probability will greatly help us to guide empirical antimicrobial treatment while awaiting cultures.

\section{List Of Abbreviations}

UTI: urinary tract infection; ESBL: Extended-Spectrum Beta-Lactamase; AAP: American Academy of Pediatrics.

\section{Declarations}

\section{Ethical Approval and Consent to participate}

Not applicable 


\section{Consent for publication}

Not applicable

\section{Availability of data and materials}

The datasets generated and/or analysed during the current study are not publicly available due privacy reasons but are available from the corresponding author on reasonable request.

\section{Competing interests}

The authors declare that they have no competing interests.

\section{Funding}

This work was supported by the National Natural Science Foundation of China (N0.81873828 NO.81771566).

\section{Authors' contributions}

Yi Wei and Shengde Wu conceived and designed the study, advised on the search, read and analysed documents, and drafted the paper. Chengjun Yu, Tianxing Zhao and Tao Lin conducted the document search, Guanghui Wei and Dawei He read and analysed the documents, and revised the manuscript. All authors take responsibility for the content of the paper.

\section{Acknowledgements}

Not applicable

\section{References}

[1] Montini G, Tullus K, Hewitt I. Febrile urinary tract infections in children. N Engl J Med. 2011. 365(3): 239-50.

[2] Shaikh N, Mattoo TK, Keren R, et al. Early Antibiotic Treatment for Pediatric Febrile Urinary Tract Infection and Renal Scarring. JAMA Pediatr. 2016. 170(9): 848-54.

[3] Hanna-Wakim RH, Ghanem ST, El HMW, et al. Epidemiology and characteristics of urinary tract infections in children and adolescents. Front Cell Infect Microbiol. 2015. 5: 45.

[4] Zorc JJ, Kiddoo DA, Shaw KN. Diagnosis and management of pediatric urinary tract infections. Clin Microbiol Rev. 2005. 18(2): 417-22.

[5] Moxon CA, Paulus S. Beta-lactamases in Enterobacteriaceae infections in children. J Infect. 2016. 72 Suppl: S41-9. 
[6] Flokas ME, Detsis M, Alevizakos M, Mylonakis E. Prevalence of ESBL-producing Enterobacteriaceae in paediatric urinary tract infections: A systematic review and meta-analysis. J Infect. 2016. 73(6): 547-557.

[7] Sorlozano A, Jimenez-Pacheco A, de Dios Luna Del Castillo J, et al. Evolution of the resistance to antibiotics of bacteria involved in urinary tract infections: a 7-year surveillance study. Am J Infect Control. 2014. 42(10): 1033-8.

[8] Mandal A, Sahi PK. Antimicrobial susceptibility of uropathogens isolated from Cambodian children. Paediatr Int Child Health. 2017. 37(3): 233.

[9] Topaloglu R, Er I, Dogan BG, et al. Risk factors in community-acquired urinary tract infections caused by ESBL-producing bacteria in children. Pediatr Nephrol. 2010. 25(5): 919-25.

[10] Dayan N, Dabbah H, Weissman I, Aga I, Even L, Glikman D. Urinary tract infections caused by community-acquired extended-spectrum beta-lactamase-producing and nonproducing bacteria: a comparative study. J Pediatr. 2013. 163(5): 1417-21.

[11] Megged O. Extended-spectrum beta-lactamase-producing bacteria causing community-acquired urinary tract infections in children. Pediatr Nephrol. 2014. 29(9): 1583-7.

[12] Uyar AN, Ekinci Z, Dundar D, Baydemir C. Childhood urinary tract infection caused by extendedspectrum beta-lactamase-producing bacteria: Risk factors and empiric therapy. Pediatr Int. 2017. 59(2): 176-180.

[13] Uwaezuoke SN, 0000-0002-2464-6645 AO, Ndu IK, Eze IC. The prevalence and risk of urinary tract infection in malnourished children: a systematic review and meta-analysis. BMC Pediatr. 2019. 19(1): 261.

[14] Ahmed M, Moremi N, Mirambo MM, et al. Multi-resistant gram negative enteric bacteria causing urinary tract infection among malnourished underfives admitted at a tertiary hospital, northwestern, Tanzania. Ital J Pediatr. 2015. 41: 44.

[15] Shin HR, Moon J, Lee HS, et al. Increasing prevalence of antimicrobial resistance in urinary tract infections of neurological patients, Seoul, South Korea, 2007-2016. Int J Infect Dis. 2019. 84: 109-115.

[16] Mori R, Lakhanpaul M, Verrier-Jones K. Diagnosis and management of urinary tract infection in children: summary of NICE guidance. BMJ. 2007. 335(7616): 395-7.

[17] Ladhani S, Gransden W. Increasing antibiotic resistance among urinary tract isolates. Arch Dis Child. 2003. 88(5): 444-5.

[18] Flores-Mireles AL, Walker JN, Caparon M, Hultgren SJ. Urinary tract infections: epidemiology, mechanisms of infection and treatment options. Nat Rev Microbiol. 2015. 13(5): 269-84. 
[19] Ramakrishnan K, Scheid DC. Diagnosis and management of acute pyelonephritis in adults. Am Fam Physician. 2005. 71(5): 933-42.

[20] Sorlozano-Puerto A, 0000-0003-0249-2291 AO, Gomez-Luque JM, et al. Etiological and Resistance Profile of Bacteria Involved in Urinary Tract Infections in Young Children. Biomed Res Int. 2017. 2017: 4909452.

[21] Saadeh SA, Mattoo TK. Managing urinary tract infections. Pediatr Nephrol. 2011. 26(11): 1967-76.

[22] Andreu A, Alos JI, Gobernado M, Marco F, de la Rosa M, Garcia-Rodriguez JA. [Etiology and antimicrobial susceptibility among uropathogens causing community-acquired lower urinary tract infections: a nationwide surveillance study]. Enferm Infecc Microbiol Clin. 2005. 23(1): 4-9.

[23] Moya-Dionisio V, Diaz-Zabala M, Ibanez-Fernandez A, et al. [Uropathogen pattern and antimicrobial susceptibility in positive urinary cultures isolates from paediatric patients]. Rev Esp Quimioter. 2016. 29(3): 146-50.

[24] Stein R, Dogan HS, Hoebeke P, et al. Urinary tract infections in children: EAU/ESPU guidelines. Eur Urol. 2015. 67(3): 546-58.

[25] Rupp ME, Fey PD. Extended spectrum beta-lactamase (ESBL)-producing Enterobacteriaceae: considerations for diagnosis, prevention and drug treatment. Drugs. 2003. 63(4): 353-65.

[26] Bruchet N, AUID- Oho, Gaschignard J, AUID- Oho, Timsit S, Cheron G. Risk of recurrence in children with a urinary tract infection due to extended-spectrum beta-lactamase producing Enterobacteriaceae. Acta Paediatr. 2020 .

[27] Albaramki JH, AUID- Oho, Abdelghani T, et al. Urinary tract infection caused by extended-spectrum beta-lactamase-producing bacteria: Risk factors and antibiotic resistance. Pediatr Int. 2019. 61(11): 11271132.

[28] Tullos JB, AUID- Oho, Stoudenmire LL, Pouliot JD. Piperacillin-Tazobactam Versus Carbapenems for the Treatment of Nonbacteremic Urinary Tract Infections due to Extended-Spectrum Beta-LactamaseProducing Enterobacteriaceae. Hosp Pharm. 2020. 55(1): 44-49.

[29] Poisson SN, Johnston SC, Josephson SA. Urinary tract infections complicating stroke: mechanisms, consequences, and possible solutions. Stroke. 2010. 41(4): e180-4.

[30] Kumar M, Dutta R, Saxena S, Singhal S. Risk Factor Analysis in Clinical Isolates of ESBL and MBL (Including NDM-1) Producing Escherichia coli and Klebsiella Species in a Tertiary Care Hospital. J Clin Diagn Res. 2015. 9(11): DC08-13.

[31] Karam G, Chastre J, Wilcox MH, Vincent JL. Antibiotic strategies in the era of multidrug resistance. Crit Care. 2016. 20(1): 136. 
[32] Sakran W, Smolkin V, Odetalla A, Halevy R, Koren A. Community-acquired urinary tract infection in hospitalized children: etiology and antimicrobial resistance. A comparison between first episode and recurrent infection. Clin Pediatr (Phila). 2015. 54(5): 479-83.

[33] Awais M, Rehman A, Baloch NU, Khan F, Khan N. Evaluation and management of recurrent urinary tract infections in children: state of the art. Expert Rev Anti Infect Ther. 2015. 13(2): 209-31.

[34] Stephens GM, Akers S, Nguyen H, Woxland H. Evaluation and management of urinary tract infections in the school-aged child. Prim Care. 2015. 42(1): 33-41.

[35] Dore-Bergeron MJ, Gauthier M, Chevalier I, McManus B, Tapiero B, Lebrun S. Urinary tract infections in 1- to 3-month-old infants: ambulatory treatment with intravenous antibiotics. Pediatrics. 2009. 124(1): 16-22.

[36] Gauthier M, Chevalier I, Sterescu A, Bergeron S, Brunet S, Taddeo D. Treatment of urinary tract infections among febrile young children with daily intravenous antibiotic therapy at a day treatment center. Pediatrics. 2004. 114(4): e469-76.

[37] Edlin RS, Shapiro DJ, Hersh AL, Copp HL. Antibiotic resistance patterns of outpatient pediatric urinary tract infections. J Urol. 2013. 190(1): 222-7.

\section{Tables}

Table 1. Risk factors for acquisition of ESBL producing UTI ( $n=480)$ 
Risk factors

Total $(\mathrm{n}=519)$

Patients with congenital urological

Malformations $\mathrm{n}=381$

Patients with no urological malformations $n=138$

Patients with vesicoureteral reflux $n=102$

Patients with no vesicoureteral reflux $n=417$

Patients with urodynamic change $n=38$

Patients with no urodynamic change $n=481$

Patients with neurologic disorder $n=32$

Patients with no neurologic disorder $n=487$

Patients with undernourishment

Patients with no undernourishment

Age $<12$ months $n=241$

Age $\geqq 12$ months $n=278$

Side of the lesion/Bilateral $n=252$

Side of the lesion/Left $n=162$

Side of the lesion/Right $n=105$

Male sex $n=334$

Female sex $n=185$

Fever $\mathrm{n}=75$

No fever $n=444$

Previous UTI in the last three months $n=241$

No UTI in the last three months $n=278$

Previous antibiotics administration in the last three months $\mathrm{n}=285$

No antibiotics administration in the last three months $n=234$

ESBL ESBL X2 P-value

$(-) \quad(+)$

$\mathrm{n}(\%) \quad \mathrm{n}(\%)$

$142 \quad 377$

$89292 \quad 9.79 \quad \mathrm{P}=0.002^{*}$

$52 \quad 86$

\begin{tabular}{llll}
19 & 83 & 4.158 & $\mathrm{P}=0.041^{\star}$ \\
122 & 295 & & \\
10 & 28 & 0.000 & $\mathrm{P}=1.000$ \\
131 & 350 & & \\
\hline & & &
\end{tabular}

80


Table 2. Logistic regression analysis of risk factors for acquisition of ESBL producing UTI

OR $(95 \% \mathrm{Cl}) \quad$ P-value

Presence of underlying neurologic disorder

\begin{tabular}{lll}
\hline No & 1 & \\
\hline Yes & $8(1.845-34.695)$ & $<0.01$ \\
\hline
\end{tabular}

Previous antibiotics administration in the last three months

No

Yes

Figures
1

$4.764(3.114-7.289) \quad<0.01$ 


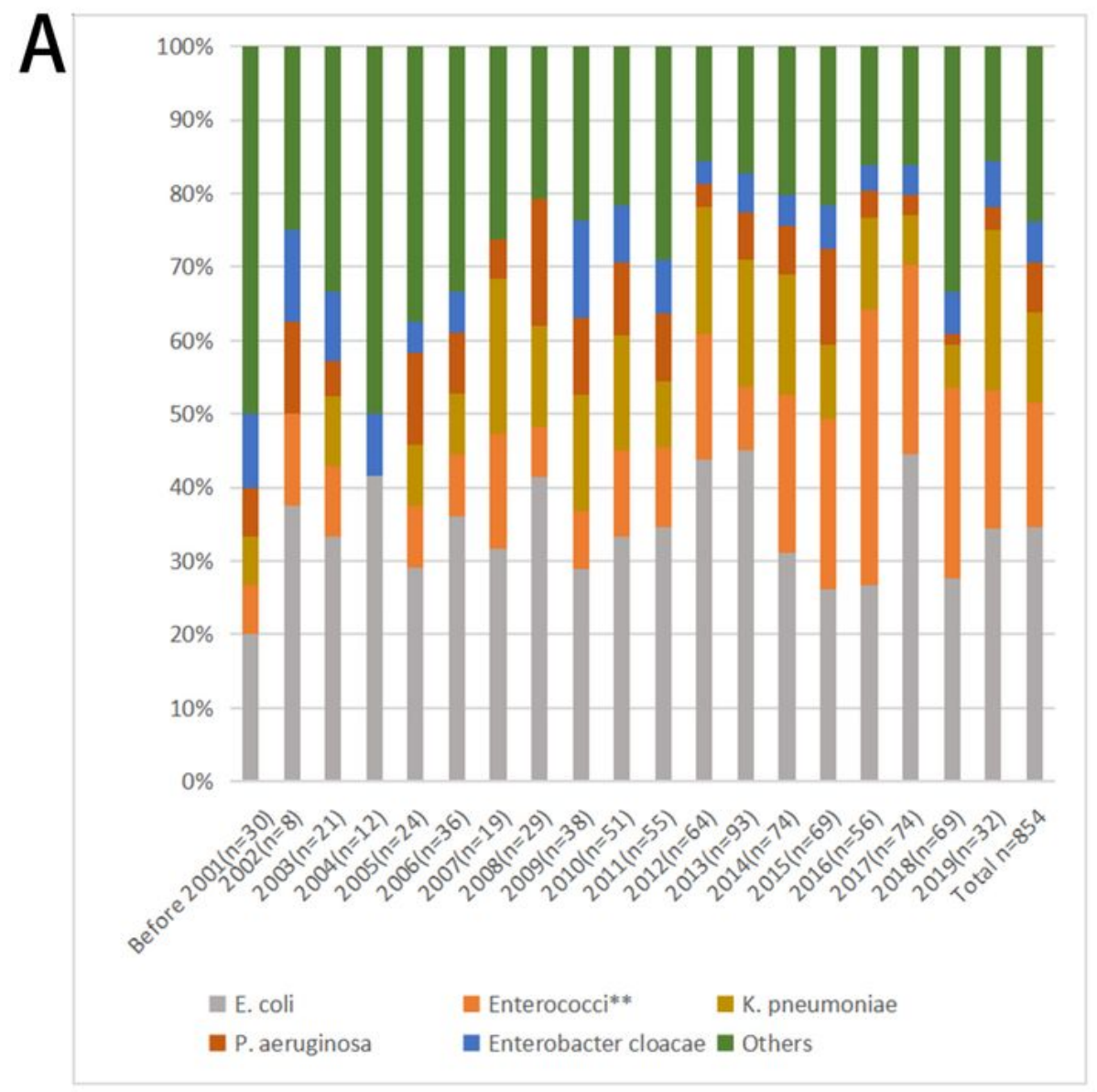

B

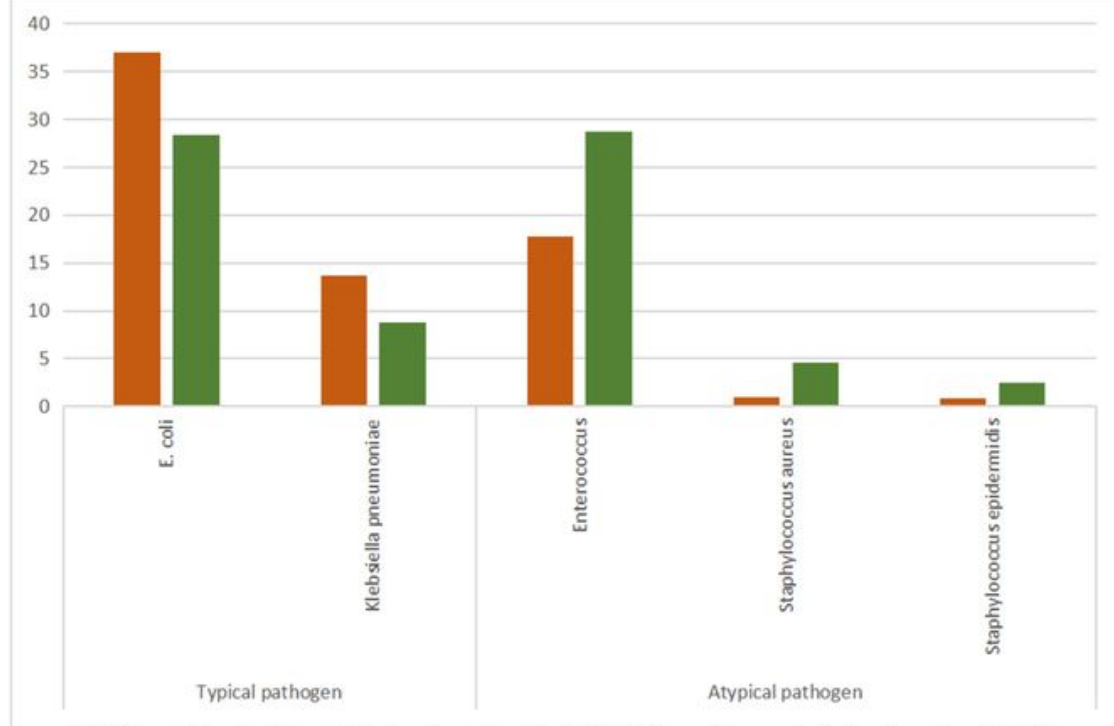

- Children with underlying urological malformations $(n=614)$ C Children with no urological malformations $(n=240)$

\section{Figure 1}

The prevalence of cultured microorganism in urinary tract infection between 1994 and 2019 The microbial etiology of urinary tract infections over the last decade were reviewed (Fig 1. A). The most common pathogen was Escherichia coli (295 cases, 34.54\%), followed by Enterococcus species (145 cases, $16.98 \%$ ), Klebsiella pneumoniae (105 cases, 12.3\%) and Pseudomonas aeruginosa (58 cases, $6.79 \%)$. The proportion of UTI cases attributed to Enterococci increased significantly ( $p=0.000)$, however, 
the overall proportion of E. coli, K. pneumoniae and P. aeruginos did not change ( $p>0.05)$. The isolated bacteria of urinary tract infections among children with and without underlying urological malformations was reviewed (Fig 1. B). The prevalence of E. coli and Klebsiella pneumoniae in children without urological abnormality was $36.97 \%$ and $13.68 \%$, which is higher than that of children with urological abnormality (28.33\% and $8.75 \%$ ). However, the prevalence of "atypical" bacteria (Staphylococcus aureus, Staphylococcus epidermidis and Enterococcus) is lower ( $17.75 \%$ vs $28.75 \%, 0.98 \%$ vs $4.58 \%, 0.81 \%$ vs $8.5 \%)$.

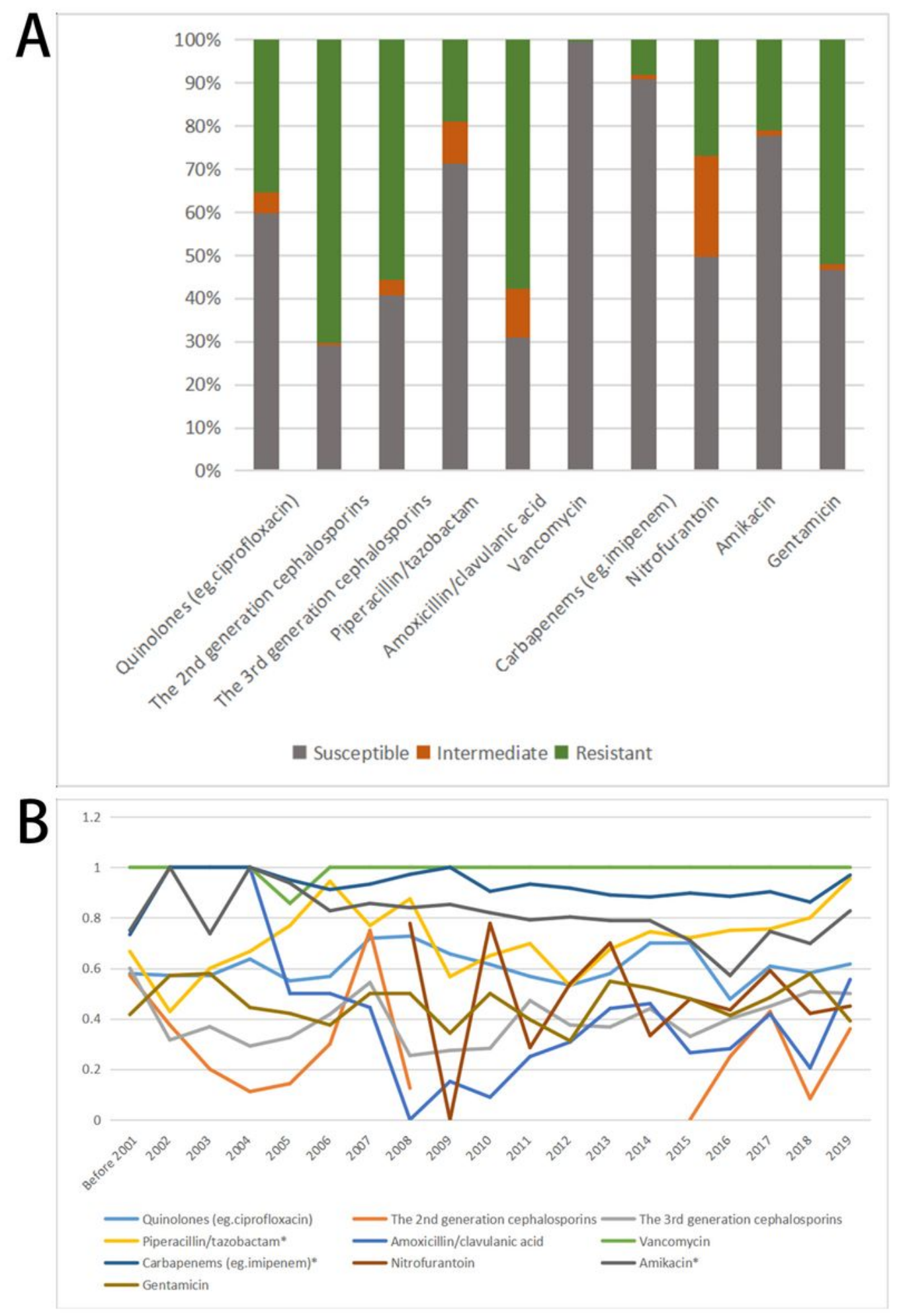




\section{Figure 2}

Susceptibility and resistance trends of commonly-prescribed antibiotics for UTI in the urology ward between 1994 and 2019 The overall susceptibility and trend of susceptibility to commonly-prescribed antibiotics were reviewed. (Figure 2. A) The overall susceptibility was relatively high to vancomycin (99.46\%) and imipenem (90.79\%) and relatively low to the 2nd generation cephalosporins (29.13\%), amoxicillin/clavulanic acid (30.99\%), the 3rd generation cephalosporins (40.64\%), nitrofurantoin (49.65\%) and gentamicin (46.51\%). The trend of susceptibility to commonly- prescribed antibiotics for UTI in the urology ward was analyzed (Figure 2. B). The susceptibility of piperacillin/tazobactam increased and the susceptibility of carbapenems decreased over the last 26 years ( $p$-value $<0.05)$. However, the susceptibility to quinolones ( $p$-value $=0.833$ ), the 3 rd generation cephalosporins ( $p$-value $=0.172$ ) and nitrofurantoin ( $p$-value $=0.246)$ did not have a significant trend over the last 26 years. 


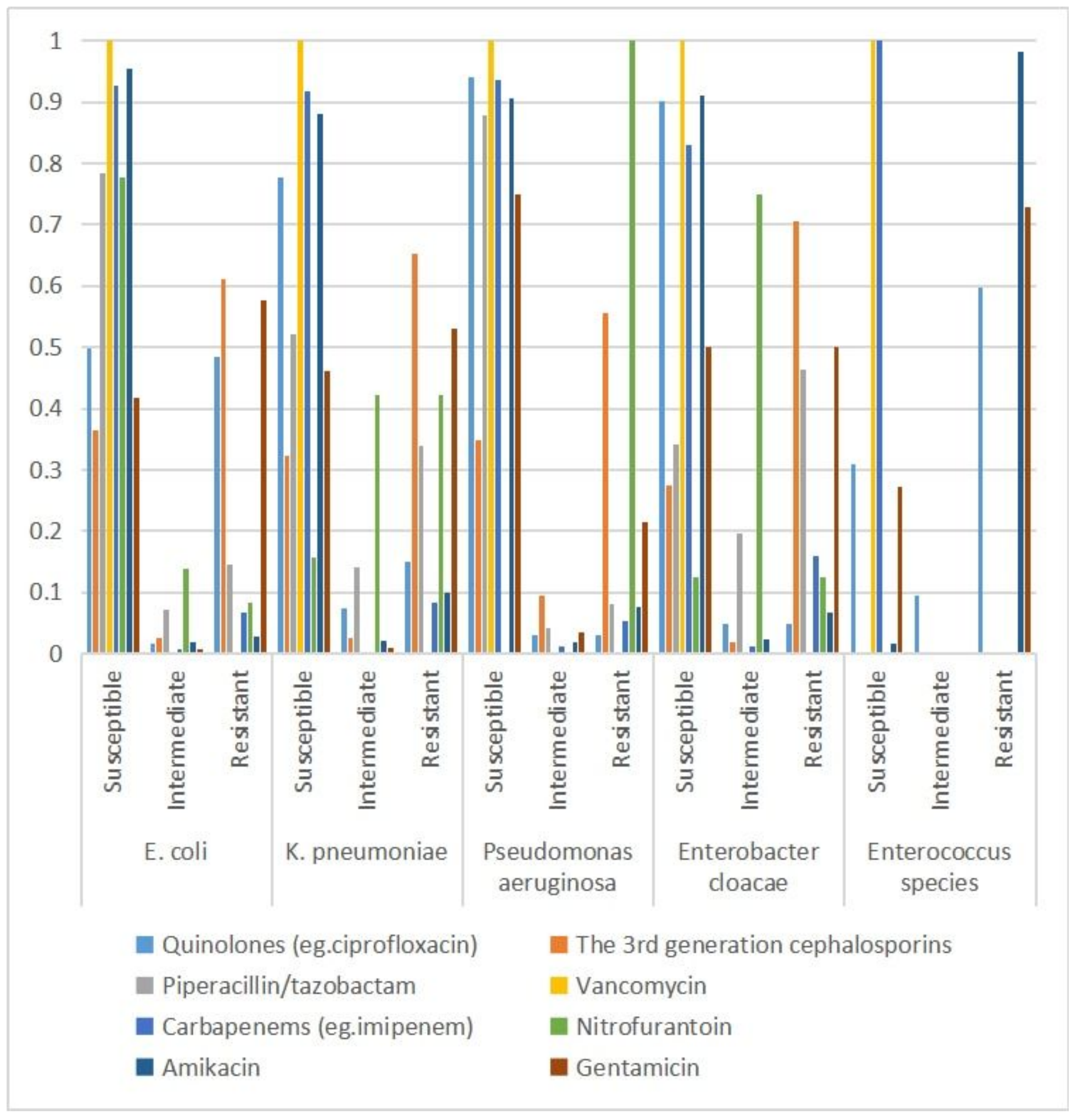

Figure 3

Antibiotic Susceptibility of the Isolated Bacteria (E. coli, K. pneumoniae, Pseudomonas aeruginosa, Enterobacter cloacae and Enterococcus species). All the isolated bacteria showed high susceptibility to carbapenems, vancomycin, and high resistance to the 3rd generation cephalosporins. E. coli showed high susceptibility to piperacillin/tazobactam $(78.34 \%)$ and nitrofurantoin $(77.78 \%)$. K. pneumoniae showed high susceptibility to quinolones (77.61\%); however, resistance to piperacillin/tazobactam and 
nitrofurantoin was recorded in at least $30 \%-40 \%$ of $\mathrm{K}$. pneumoniae isolates in these years. Enterobacter cloacae were also detected high resistance to piperacillin/tazobactam with resistance rates $>45 \%$.

Pseudomonas aeruginosa showed high susceptibility to quinolones (93.94\%) and piperacillin/tazobactam (87.76\%), with annual resistance rates $<15 \%$. Enterococcus species showed high susceptibility to carbapenems and vancomycin, whereas resistance to gentamicin, amikacin, and quinolones.
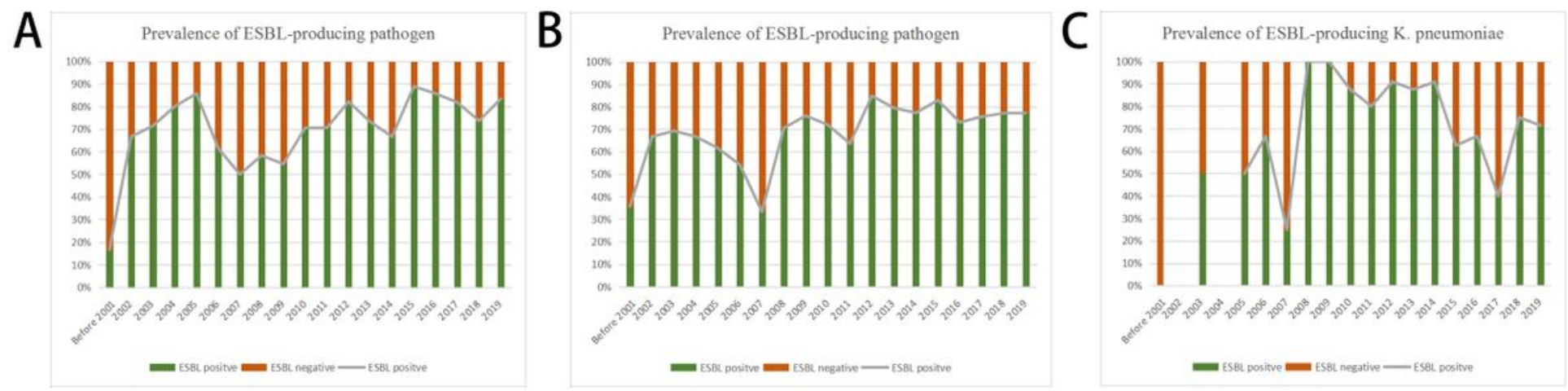

\section{Figure 4}

Trend of antibiotics resistance in UTI between 1994 and 2019. We assessed the changing trends of extended-spectrum beta-lactamase (ESBL)-producing pathogens in E. coli and K. pneumoniae, which are the most common in UTI. (A)Among the whole isolated bacteria in UTIs, the proportion of ESBL-producing pathogens increased significantly ( $p$-value $=0.003)$, (B) Among E. coli UTI, there was an observed increase in the proportion of ESBL producing pathogens ( $p$-value $=0.021)$, (C) Among the UTIs exhibiting a microbial etiology attributed to K. pneumoniae, the proportion of ESBL-producing pathogens did not increase ( $p$-value $=0.482$ ). 


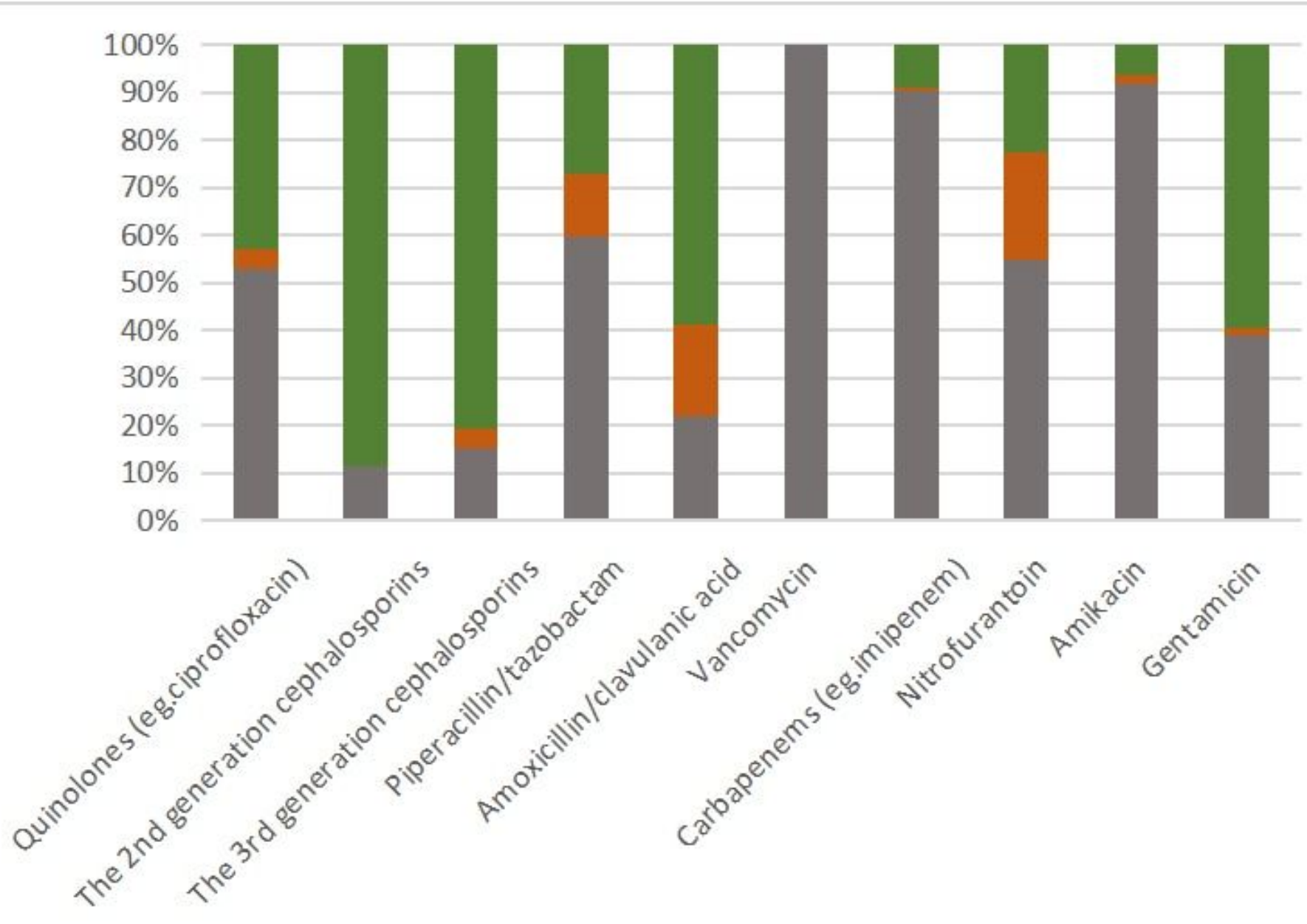

Susceptible $\mathbf{a}$ Intermediate $\mathbf{a}$ Resistant

Figure 5

Susceptibility and resistance trends of commonly- prescribed antibiotics for ESBL-UTI. The overall susceptibility and trend of susceptibility to commonly- prescribed antibiotics were reviewed. (A) The overall susceptibility was relatively high to vancomycin (100.00\%), carbapenems $(90.10 \%)$, amikacin (91.90\%) and relatively low to the 2nd generation cephalosporins (11.22\%), the 3rd generation cephalosporins (15.18\%) and amoxicillin/clavulanic acid (21.86\%). 
A

Points

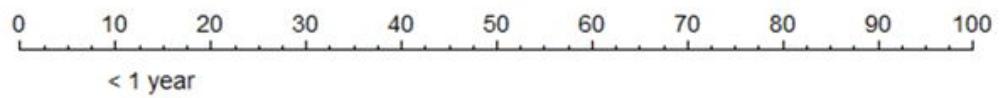

Age

UTI

Fever

Neurologic disorders

Antibiotics administration

Renal anomalies

Vesicoureteral reflux

Malnourished

Total Points

ESBL rate
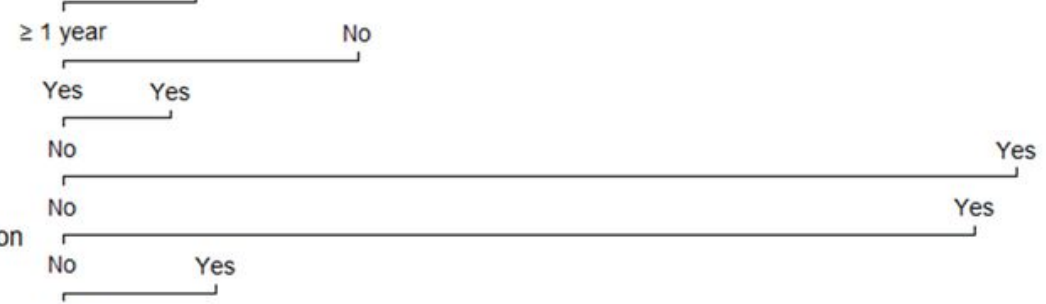

No Yes
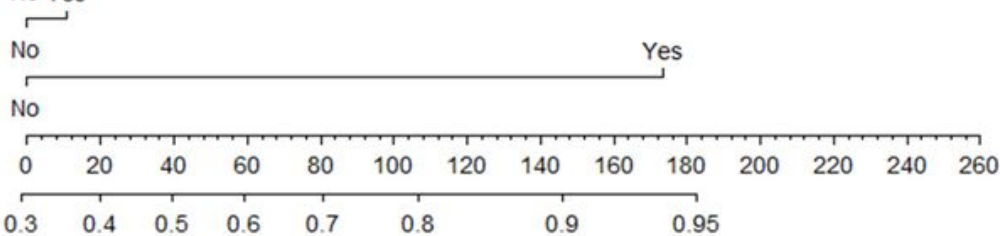

B

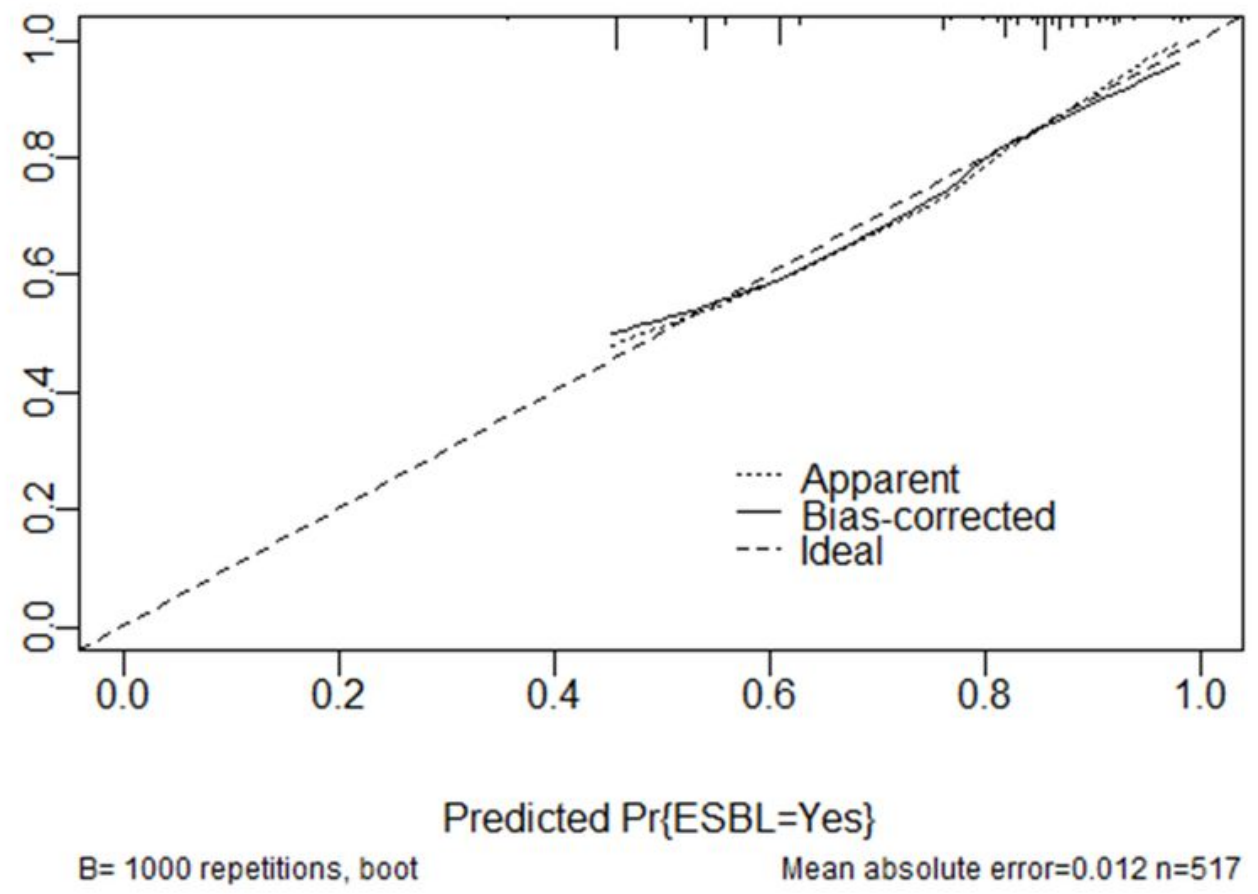

Figure 6

Nomogram to predict ESBL (+) UTI (Fig 6.A) and calibration plot for predicting ESBL (+) UTI (Fig 6.B). 